\title{
Reserpine Improves Working Memory
}

\author{
Raghuraman Vasantharaja' ${ }^{1}$, Ajeet Kumar ${ }^{1}$, Ashok Kumar ${ }^{1}$, Jamuna R. Subramaniam 1,2* \\ ${ }^{1}$ Department of Biological Sciences and Bioengineering, Indian Institute of Technology, Kanpur, India \\ ${ }^{2}$ Center for Preclinical and Translational Medicine Research, Central Research Facility, Sri Ramachandra \\ University, Chennai, India

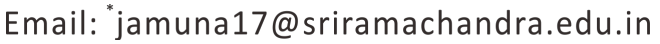

Received 15 December 2015; accepted 8 March 2016; published 11 March 2016

Copyright (C) 2016 by authors and Scientific Research Publishing Inc.

This work is licensed under the Creative Commons Attribution International License (CC BY). http://creativecommons.org/licenses/by/4.0/

(c) O) Open Access

\begin{abstract}
Despite exhaustive search, no drug is in sight for AD. Earlier, we reported that reserpine, an antihypertensive and antipsychotic drug, ameliorates Amyloid beta ( $A \beta$-AD causing peptide) toxicity and confers several positive enhancements in the $C$. elegans model system. Here, we evaluate whether reserpine can provide protection against working memory and against AD in the mouse model. Reserpine $(0.08 \mathrm{mg})$ was administered orally on alternate days to the non- $\mathrm{Tg}$ and accelerated $A \beta$ deposition (at 2 months of age)and cognitive deficit (4 months of age) developing 5XFAD AD Tg mouse model expressing mutant human APP (3 familial mutations) and human Presenilin1( 2 familial mutations) in the neurons, and follow their working memory for 2 months using the spontaneous Y-maze alteration behavioral paradigm. Reserpine enhanced working memory in non-Tg mice and improved the cognitive deficit in the 5XFAD AD Tg mice. Hence, reserpine can be considered for a detailed evaluation in the $3 X \mathrm{Tg}$ AD mouse model and a pilot study in AD patients.
\end{abstract}

\section{Keywords}

Alzheimer's Disease, Amyloid- $\beta$-A $\beta$, Reserpine, Cognitive Deficits, Transgenic Mice, Working Memory

\section{Introduction}

Alzheimer's disease, the devastating neurodegenerative disease occurring at epidemic proportions (1 in 85 people) is predicted to reach $\sim 115.4$ million people in 2050 [1]. Despite screening $1000 \mathrm{~s}$ of drugs to treat AD [2] [3], any worthwhile drug is yet to come. Rather, many drugs, immunization with $\mathrm{A} \beta$ [4] for its clearance and $\mathrm{A} \beta$ generating gamma and beta-secretase inhibitors hardly showed any protection in clinical trials. So a potential treatment for AD is an urgent necessity.

A $\beta$ neuritic plaques [5] and phospho-tau neurofibrillary tangles [6] [7], loss of synapses and neurons in the brain leading to cognitive deficits-progressive dementia and eventually death were observed in AD patients [8].

\footnotetext{
${ }^{*}$ Corresponding author.
} 
Cholinergic nervous system is impaired and lost as the disease progresses. The glutamate NMDA receptor is involved in learning and memory by long term potentiation and synaptic plasticity. A modest protection against moderate and severe AD is brought about by the (NMDA) inverse antagonist memantine ([2] and references within). Acetylcholine esterase inhibitors are the more common treatment which provides minimal delay of the symptoms, but extremely expensive [2].

Majority of AD cases are sporadic. But accelerated familial AD development is noticed in patients with mutations in two genes, namely, Amyloid Precursor Protein (APP) and Presenilin 1 ([6] and references within). Several AD transgenic mice models [9] have been developed with these mutations to understand the mechanism of $\mathrm{AD}$ and for the development/identification of drugs, with limited success. Earlier models lacked neuronal loss found in AD patients. To fill in this gap, Oakley et al. [10] have developed a model, which expresses 5 different mutations of human APP and Presenilin 1 namely, three familial mutations (K670N/M671L (Swedish), I716V (Florida), and V717I (London)) in APP and two mutations (PS1 M146L and L286V) in Presenilin 1(PS1). These 5XFAD AD Tg mice start showing $\mathrm{A} \beta$ aggregates from 2 months of age [10]. They display progressive neuronal loss and behavioral deficits [10], the two most important features in AD patients. Hence, we chose this model for our studies.

Antihypertensive drugs provide protection against Dementia. A screen of many antihypertensive drugs to reduce $\mathrm{A} \beta$ aggregates in a cell culture model led to the identification of Valsaratan which was later proved to provide protection in AD model mice [11], which is currently in clinical trials [11]. The Cache County study suggests that patients taking antihypertensive drugs are less likely to develop Alzheimer's disease [12].

Reserpine is a FDA approved antihypertensive drug from the roots of Rauwolfia serpentina, known as Sarpaganda being used in ayurvedic medicine in India for 1000s of years [13] to treat insanity, snake bites and as a tranquilizer. Independently, we identified that reserpine could provide high quality lifespan extension in C. elegans [14]. In addition, reserpine ameliorates $\mathrm{A} \beta$-induced toxicity manifested as progressive paralysis, confered stress tolerance and enhanced locomotion till late age in the $C$. elegans model [15]. The AD mouse model, $\mathrm{Tg} 2576$, expresses human mutant APP, which causes A $\beta$ aggregates formation and cognitive deficits at $\sim 11$ months of age [16]. In these Tg2576 mice, reserpine reduced $\mathrm{A} \beta 42$ deposits and levels in hippocampus and serum [16]. But reserpine's effect on the major problem of $\mathrm{AD}$, namely, cognitive deficit—progressive dementia or memory loss was not addressed.

Here, we address whether reserpine: 1) provides protection against $\mathrm{A} \beta$ toxicity induced cognitive deficits in the 5XFAD AD Tg model and 2) improves cognition in normal mice.

\section{Materials and Methods}

\subsection{Animals and Maintenance}

The mice were maintained in standard animal house facility with ad libitum access to food and water. All animal protocols were approved by the Institutional Animal Ethics Committee and in accordance with the guidelines of CPCSEA. The 5X FAD AD Tg mice [10] (obtained from Jackson laboratory, Ann Arbor, USA) were bred with wild-type non-transgenic mice to yield transgenic and non-transgenic littermates and genotyped using the standard tail DNA genomic PCR with relevant primer sets sets (APP: APP-FP (AGGACTGACCACTCGACCAG) \& APP-RP (CGGGGGTCTAGTTCTGCAT); Presenilin1: PS1-FP (AATAGAGAACGGCAGGAGCA) \& PS1 -RP (GCCATGAGGGCACTAATCAT)).

\subsection{Reserpine Treatment}

Reserpine (Sigma) (0.08 mg), dissolved in fruit vinegar and diluted with dextrose-saline was orally administered to human ${ }_{\mathrm{m}} a p p_{\mathrm{m}}^{+}$psen $1^{+}$(5XFAD)/non-transgenic mice on alternate days from 2 months of age till 5 months. This dosage and duration was arrived at based on the specific concentration at which reserpine had protective effects in C. elegans [14] [15] and the prescription used for hypertension [13]. For control, the vehicle, fruit vinegar, diluted with saline dextrose was administered. All the mice were age and sex matched. The treatment was done as three independent sets, with 4 or 5 mice per group with approximately equal number of males and females.

\subsection{Behavioral Analyses}

After one month of drug regimen, spatial working memory was tested with minor modifications [17]. This 
learning task assesses hippocampus dependent spatial learning, working memory and exploratory activity. Mice were individually placed in the center of the symmetrical Y-maze consisting of three arms separated by 120 degrees (arm size: $40 \mathrm{~cm}$ long; $13 \mathrm{~cm}$ high and $10 \mathrm{~cm}$ wide). Mice were allowed to explore freely through the Ymaze during a $5 \mathrm{~min}$ session. The sequence of arms entered and total number of arms entered were recorded. Number of arm entry was considered when all the four paws were placed inside the arm. This behavior was evaluated on alternate days starting from 3 months up to 5 months of age, since the 5XFAD Tg mice shows poor performance of spontaneous alternation from the age of $\sim 4$ months due to cognitive decline as described previously [10]. The number of triads was calculated as 3 sequential entries into three different arms (i.e. 1, 2, 3 or $2,3,1)$.

\subsection{Statistical Analysis}

The data obtained was analyzed using SigmaPlot version 10.0 and the statistical significance was performed by student's $t$ test. All results were expressed as mean \pm standard deviation. P-value $<0.01$ and P-value $<0.001$ were considered to be statistically significant.

\section{Results}

Reserpine, an antihypertensive drug was protective in the $\mathrm{A} \beta$ toxicity model in $C$. elegans. Further, reserpine reduced $\mathrm{A} \beta$ levels in the Tg2576 AD model mice. Here, we evaluated the crux of the disease, improvement from the cognitive deficit impairment, measured using the behavioral paradigm-spontaneous alteration in the $\mathrm{Y}$ maze for working memory in the 5 XFAD AD Tg mouse model. They develop cerebral A $\beta$ plagues (aggregates) rapidly at two months of age and show memory impairment in Y maze by 4 months of age. Both APP and PS1 segregate together $[10]$ and the mice with these genes were identified.

\subsection{Reserpine Delays Cognitive Deficits in the 5XFAD AD Tg Mice}

In order to determine reserpine's ability to alleviate $\mathrm{A} \beta$ toxicity in the mouse model, in which the actual behavioral deficit in cognition could be determined, we orally administered the 5 XFAD AD Tg mice with $\sim 0.08 \mathrm{mg}$ of reserpine on alternate days from two months of age up to 5 months of age. We followed the improvement in the cognitive paradigm, spontaneous alteration behavior task in the Y-maze on alternate days, usually on the next day after reserpine oral administration. The behavioral paradigm evaluation was started after one month of initiation of drug regimen and monitored for 2 months which is up to 5 months of age.

In the Y-maze paradigm, overall total movement and spontaneous arm alteration counted as triad (entry into all the three arms in a sequential manner) were measured in the 5X FAD AD Tg mice. Each trial lasted for 5 minutes. Reserpine increases the overall movement and the maximum activity is observed after one month of initiation of behavioral paradigm. The actual measure of cognitive enhancement/protection measured as increase in the number of triads entered also peaks after 20 days of start of the Y-maze behavioral paradigm. Of these, $\sim 8$ days (118 - 126 days of age) (Figure 1$)$ showed the maximum protection which was statistically significant $(\mathrm{P}<$ $0.005 / \mathrm{P}<0.05)$.

\subsection{Reserpine Treatment Enhances Cognition in the Normal Mice}

When normal non-Tg mice were treated with the same dose of reserpine as the human mutant $\operatorname{app}^{+}$psen $1^{+}$mice they showed improvement in cognition as number of triads entered (Figure 2). Moreover, the enhancement was statistically significant ${ }^{* *}-\mathrm{P}<0.005$ and ${ }^{*}-\mathrm{P}<0.05$ ) for a long duration of almost one month (Figure 2). Thus, reserpine was able to enhance normal cognition as well.

\section{Discussion}

The 5XFAD AD Tg mouse model showed enhanced spatial working memory measured by increase in the number of triads entered when they were chronically administered with low dosage $(0.08 \mathrm{mg})$ reserpine orally on alternate days for a period of three months. More importantly, reserpine improved the cognition of the 5xFAD AD Tg mice at the age (Figure 1) where these mice are reported to show cognitive deficits. While our study was ongoing, Go et al. [16] reported that reserpine can help relieve AD pathogenesis in Tg2576 mice through down 


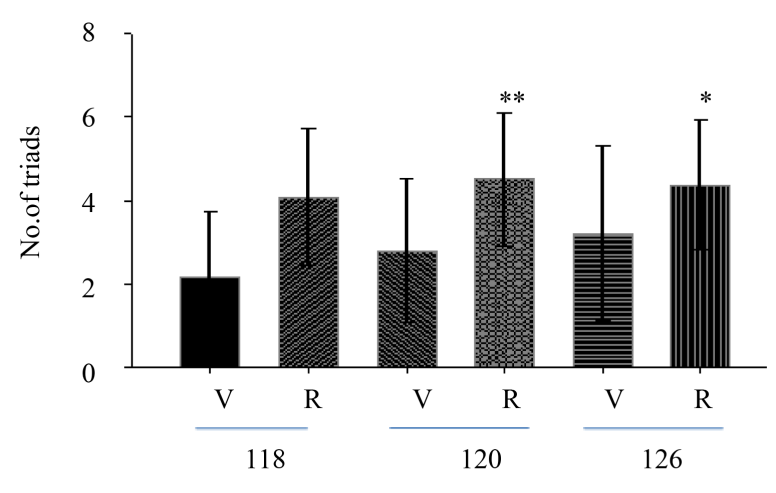

Age in days

Figure 1. Reserpine increasing cognition in the 5XFAD AD Tg mice. Number of triads entered by V-Vehicle $(n=14)$ and R-reserpine treated $(n=14)$ 5XFAD Tg mice at different ages. V-Vehicle; R-Reserpine treated; ${ }^{* *}-\mathrm{P}<0.001$; $^{*}-\mathrm{P}<$ 0.01. Error bar-Standard Deviation.

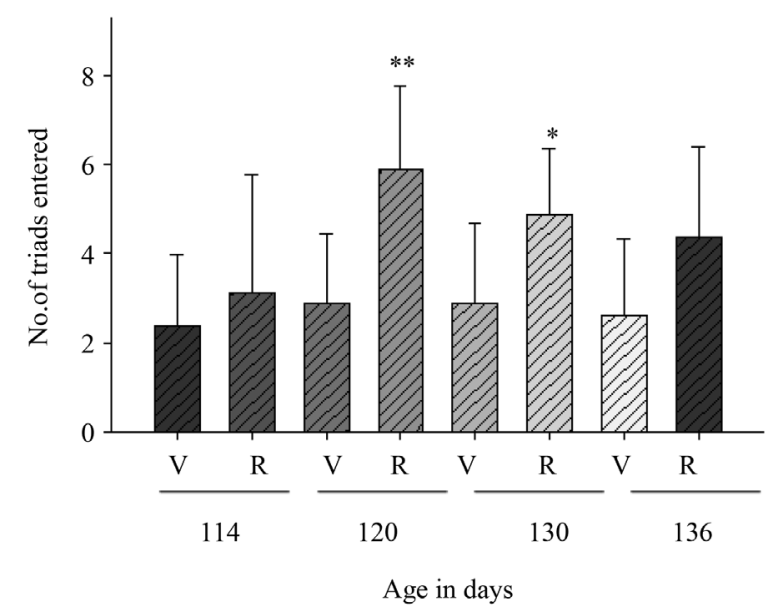

Figure 2. Reserpine improves cognitive ability in the non- $\mathrm{Tg}$ mice. The no. of triads entered by V-Vehicle $(n=14)$ and R-reserpine treated $(\mathrm{n}=14)$ non-Tg mice at different ages. ${ }^{* *}-\mathrm{P}<0.001 ;{ }^{*}-\mathrm{P}<0.01$. Error bar-Standard Deviation.

regulation of $\mathrm{A} \beta_{42}$ deposition, NGF secretion [16], but had not assessed cognitive function. Since decline in cognition is the most devastating feature of $\mathrm{AD}$, here, we show that reserpine improves spatial working memory and delays cognitive deficits in the 5XFAD AD Tg mice at the low dosage of $0.08 \mathrm{mg}$ that too administered only on alternate days which is in line with the low dosage of reserpine currently in use. Reserpine is effective as an antihypertensive drug at as low a dosage as $0.05 \mathrm{mg}$ [13] [18]. In addition, reserpine enhances cognition in the normal mice (Figure 2). This suggests that reserpine can be considered for detailed evaluation in the $3 \mathrm{X} \mathrm{Tg}$ (human mutant APP, mutant Presenilin 1 and mutant tau) AD mouse model and a pilot screen in AD patients.

In addition to dementia, due to the progressive neuronal loss, other complications like anxiety, paranoia, aggression, hallucinations, delusions, and depression and sleep disturbances make management of AD patients extremely difficult. As reserpine modulates biogenic amines neurotransmitters, the secondary problems mentioned above also could be alleviated by it. Go et al. [16], report that reserpine can significantly reduce the aggressive behavior in the Tg2576 AD mice. In Ayurvedic medicine, reserpine has been used as an antipsychotic and tranquilizer for more than a millennium.

Reserpine has a strong potential to be a candidate drug for $\mathrm{AD}$, because: 1) first and foremost, it improves working memory (Figure 1 and Figure 2); 2) it decreases A $\beta$ 42-deposits/levels in brain and serum [16]; 3) it 
increases NGF secretion and its action through TrkA signaling[16]; 4) it induces expression of antiapoptotic protein, BCL-2 which can protect against neuronal loss [16]; 5) it does not inhibit or drastically downregulate the gamma-secretase complex [16] which is crucial for notch signaling, lack of which leads to cancer [3] [4]; 6) it can cross the blood brain barrier; 7) the major caveat about reserpine is the development of depression, and at a low dosage of $1 \mathrm{mg} / \mathrm{kg}$ reserpine does not cause depression in mice and the maniac depression pathological marker mkb-1 is not induced [19], but at the dosage of $2 \mathrm{mg} / \mathrm{kg}$ both these effects are noticed [19]; 8) it is widely in use, especially as an antihypertensive drug, even as a community based prophylactic at the low dosage of $0.05 \mathrm{mg}$ against hypertension with minimal side effects for several years [13] [20]; 9) it reduces mortality when used as an antihypertensive [17].

Thus, Reserpine ameliorates $\mathrm{A} \beta$ induced cognitive deficit in the 5XFAD Tg mice at a low dosage of $0.08 \mathrm{mg}$ orally administered on alternate days. Hence, reserpine's efficacy can be evaluated in detail in the 3xTG AD model mice. In addition, reserpine analogs could be synthesized and evaluated for protection against AD. More importantly, given the limitations of animal models, exceptionally fruitful approach will be to carry out a pilot screen in AD patients given the severity, prevalence, social and financial burden on the society and the urgent need of a drug for $\mathrm{AD}$.

\section{Conclusion}

Reserpine ameliorates $\mathrm{A} \beta$ induced cognitive deficit in the 5XFAD Tg mice at a low dosage of $0.08 \mathrm{mg}$ orally administered on alternate days. This makes reserpine a potential drug to be evaluated in AD patients. As reserpine is originally identified to be protective in the $\mathrm{A} \beta$ toxicity $C$. elegans model, the $C$. elegans system is a worthwhile model for preclinical drug discovery.

\section{Acknowledgements}

The authors acknowledge the financial support from Department of Biotechnology, Government of India and Jackson laboratory, Ann Arbor USA for the 5xFAD AD Tg mice.

\section{References}

[1] Mori, T., Koyama, N., Segawa, T., Maeda, M., Maruyama, N., Kinoshita, N., Hou, H., Tan, J. and Town, T. (2014) Methylene Blue Modulates $\beta$-Secretase, Reverses Cerebral Amyloidosis, and Improves Cognition in Transgenic Mice. Journal of Biological Chemistry, 289, 30303-30317. http://dx.doi.org/10.1074/jbc.M114.568212

[2] Wollen, K.A. (2010) Alzheimer's Disease: The Pros and Cons of Pharmaceutical, Nutritional, Botanical, and Stimulatory Therapies, with a Discussion of Treatment Strategies from the Perspective of Patients and Practitioners. Alternative Medicine Review, 15, 223-244.

[3] Savonenko, A.V., Melnikova, T., Hiatt, A., Li, T., Worley, P.F., Troncoso, J.C., Wong, P.C. and Price, D.L. (2012) Alzheimer's Therapeutics: Translation of Preclinical Science to Clinical Drug Development. Neuropsychopharmacology, 37, 261-277. http://dx.doi.org/10.1038/npp.2011.211

[4] Doody, R.S., Thomas, R.G., Farlow, M., Iwatsubo, T., Vellas, B., Joffe, S., Kieburtz, K., Raman, R., Sun, X., Aisen, P.S., Siemers, E., Liu-Seifert, H., Mohs, R., Alzheimer's Disease Cooperative Study Steering Committee and Solanezumab Study Group (2014) Phase 3 Trials of Solanezumab for Mild-to-Moderate Alzheimer's Disease. The New England Journal of Medicine, 370, 311-321. http://dx.doi.org/10.1056/NEJMoa1312889

[5] Glenner, G.G. and Wong, C. (1984) Alzheimer's disease: Initial Report of the Purification and Characterization of a Novel Cerebrovascular Amyloid Protein. Biochemical and Biophysical Research Communications, 120, 885-890.

[6] Selkoe, D.J. (2001) Alzheimer's Disease: Genes, Proteins, and Therapy. Physiological Reviews, 81, 741-746.

[7] Song, M.S., Rauw, G., Baker, G.B. and Kar, S. (2008) Memantine Protects Rat Cortical Cultured Neurons against Beta-Amyloid-Induced Toxicity by Attenuating Tau Phosphorylation. European Journal of Neuroscience, 28, $1989-2002$.

[8] Lopez, O.L. and DeKosky, S.T. (2003) Neuropathology of Alzheimer's disease and Mild Cognitive Impairment. Review Neurology, 37, 155-163.

[9] Borchelt, D.R., Ratovitski, T., van Lare, J., Lee, M.K., Gonzales, V., Jenkins, N.A., Copeland, N.G., Price, D.L. and Sisodia, S. (1997) Accelerated Amyloid Deposition in the Brains of Transgenic mice Coexpressing Mutant Presenilin 1 and Amyloid Precursor Proteins. Neuron, 19, 939-945. http://dx.doi.org/10.1016/S0896-6273(00)80974-5

[10] Oakley, H., Cole, S.L., Logan, S., Maus, E., Shao, P., Craft, J., Guillozet-Bongaarts, A., Ohno, M., Disterhoft, J., Van Eldik, L., Berry, R. and Vassar, R. (2006) Intraneuronal Beta-Amyloid Aggregates, Neurodegeneration, and Neuron 
Loss in Transgenic Mice with Five Familial Alzheimer's Disease Mutations: Potential Factors in Amyloid Plaque Fomation. The Journal of Neuroscience, 26, 10129-10140. http://dx.doi.org/10.1523/JNEUROSCI.1202-06.2006

[11] Wang, J., Ho, L., Chen, L., Zhao, Z., Zhao, W., Qian, X., Humala, N., Seror, I., Bartholomew, S., Rosendorff, C. and Pasinetti, G. (2007) Valsartan Lowers Brain Beta-Amyloid Protein Levels and Improves Spatial Learning in a Mouse Model of Alzheimer Disease. Journal of Clinical Investigation. 117, 3393-3402. http://dx.doi.org/10.1172/JCI31547

[12] Khachaturian, A.S., Zandi, P.P., Lyketsos, C.G., Hayden, K.M., Skoog, I., Norton, M.C., Tschanz, J.T., Mayer, L.S., Welsh-Bohmer, K.A. and Breitner, J.C. (2006) Antihypertensive Medication Use and Incident Alzheimer Disease: The Cache County Study. Archives of Neurology, 63, 686-692. http://dx.doi.org/10.1001/archneur.63.5.noc60013

[13] Lele, R. (2010) Beyond Reverse Pharmacology: Mechanism-Based Screening of Ayurvedic Drugs. Journal of Ayurveda and Integrative Medicine, 1, 257-265. http://dx.doi.org/10.4103/0975-9476.74435

[14] Srivastava, D., Arya, U., SoundaraRajan, T., Dwivedi, H., Kumar, S. and Subramaniam, J.R. (2008) Reserpine Can Confer Stress Tolerance and Lifespan Extension in the Nematode C. elegans. Biogerontology, 9, 309-316. http://dx.doi.org/10.1007/s10522-008-9139-5

[15] Arya, U., Dwivedi, H. and Subramaniam, J.R. (2009) Reserpine Ameliorates A $\beta$ Toxicity in the Alzheimer's Disease Model in Caenorhabditis elegans. Experimental Gerontology, 44, 462-466. http://dx.doi.org/10.1016/j.exger.2009.02.010

[16] Go, J., Cho, S.I., Kim. J.I, Lee, Y.J., Kwak, M.H., Koh, E.K., Song, S.H., Sung, J.E. and Hwang, D.Y. (2013) Effect of Reserpine on the Behavioral Defects, A $\beta-42$ Deposition and NGF Metabolism in Tg2576 Transgenic Mouse Model for Alzheimer's Disease. Journal of Life Sciences, 23, 812-824. http://dx.doi.org/10.5352/JLS.2013.23.6.812

[17] Ohno, M., Chang, L., Tseng, W., Oakley, H., Citron, M., Klein, W.L., Vassar, R. and Disterhoft, J.F. (2006) Temporal Memory Deficits in Alzheimer's Mouse Models: Rescue by Genetic Deletion of BACE1. European Journal of Neuroscience, 23, 251-260. http://dx.doi.org/10.1111/j.1460-9568.2005.04551.x

[18] Curb, J.D., Schneider, K., Taylor, J.O., Maxwell, M. and Shulman, N. (1988) Antihypertensive Drug Side Effects in the Hypertension Detection and Follow-Up Program. Hypertension, 11, 1151-1155. http://dx.doi.org/10.1161/01.HYP.11.3 Pt 2.II51

[19] Lee, H.R., Hwang, I.S., Kim, J.E., Cho, S.I., Lee, Y.J., Goo, J.S., Lee, E.P., Choi, H.W., Kim, H.S., Lee, J.H., Jung, Y.J. and Hwang, D.Y. (2012) Altered Expression of $\gamma$-Secretase Components in Animal Model of Major Depressive Disorder Induced by Reserpine Administration. Laboratory Animal Research, 28, 109-114. http://dx.doi.org/10.5625/lar.2012.28.2.109

[20] Feigenbaum, L.J. (1993) Reserpine and Thiazide for Hypertension-An Inexpensive Therapy for Older Patients. Western Journal of Medicine, 158, 535. 